\title{
Research on automatic block binarization method of stained Tibetan ancient document image based on Lab color space
}

\author{
Yuehui Han ${ }^{1, a}$, Weilan Wang ${ }^{*, 2, b}$, Yiqun Wang ${ }^{3, c}$ \\ 1,2,3School of Math and Computer Science, Northwest Minzu University, LanZhou China \\ aqusthanyh@163.com, *,bwangweilan@xbmu.edu.cn, cwyq6696@gsli.edu.cn
}

\begin{abstract}
Keywords: Tibetan ancient document image, Lab color space, Stain removal, Binarization Abstract. The binarization of Tibetan ancient document image have always been the focus of research, in this paper, an automatic block local binarization method based on Lab color space is proposed for stained Tibetan ancient document image. Firstly, weaken and eliminate the influence of the stains using the location information of the stains obtained by the information of the image itself in Lab color space. Secondly, the image is automatically partitioned according to the number and size of the character in the image. Finally, local binarization processing is performed and binarized image is obtained. The experiment shows that this method has a good binarization effect to the stained Tibetan ancient document image.
\end{abstract}

\section{Introduction}

Tibetan ancient document is the rare historical materials for researching Tibetan's politics, history, culture and medicine, which is the crystallization of the wisdom of the Tibetan people in the past thousands years. Tibetan ancient document has important academic value and research significance that was highly valued by domestic and foreign experts [1]. For a long time, the Tibetan ancient document has been degraded in varying degrees because of the improper preservation conditions and the damage of natural or man-made factors. Such as color degradation causes text to be indistinct, the stamp blur caused by heavy butter stain or artificial mark, as well as ancient material itself vulnerable to corrosion, so it cannot afford to repeatedly read and use. Therefore, the digital protection and restoration of Tibetan ancient document is very important. The binarization, as a pre-processing technique, is one of the most important tasks in the digitization of Tibetan ancient document.

The image binarization can be divided into gray image binarization and color image binarization: gray image binarization is based on threshold method, which is to find a suitable threshold to segment the image into two classes (target and background) and then get the binarized image. The selection of threshold plays a decisive role in the result of image binarization. At present, image binarization methods based on threshold can be divided into global threshold method and local threshold method [2]: The global threshold method uses a single threshold for the whole image, and judges the target and the background by comparing the threshold with all pixel values. One of the widely used algorithms is the OTSU algorithm proposed by N.OTSU [3], this algorithm has strong adaptability. However, for some complex background images with larger background gray change, uneven illumination or dirty stains, the OTSU algorithm can only obtain a single threshold, and cannot take into account the actual situation of each sub region in the image. Therefore, the image cannot be effectively segmented [4]. In order to solve the problem of global threshold method, some scholars have put forward the local threshold method, the method does not use a single global threshold, but compares each point with other pixels in its local neighborhood. The threshold of each pixel is determined according to local characteristics, and then image binarization are processed. Typical local binarization algorithms are BERNSEN [5], NIBLACK[6], SAUVOLA [7] and so on. A large number of scholars found that for uneven illumination image or low contrast image, using the local threshold method can get better binarization effect, but for the stained image this method inevitably will lead to exist the false targets. Therefore, many scholars have put forward some other improvement methods [8-9], good results have been achieved, but in some cases the results are still unsatisfactory. Color image binarization [10-11] have been paid more attention in recent years, Lv 
Yanna et al [12] expounds several color spaces frequently involved in the process of digital image processing, and emphatically discusses the transformation relation of RGB and HIS color space in actual use. Yang Jing et al [13] proposed a threshold segmentation algorithm that can segment color images directly based on the features of RGB color space. Peng Xiaomin et al [14] proposed a color image segmentation method based on HSI and Lab color space. Firstly the optimum threshold method was used to segment image in HSI color space, secondly K mean clustering method was used to segment image in Lab color space, thirdly the two segmentation results are merged, finally the windowed filtering was used to eliminate noise. Jiang Jichun et al [15] proposed an algorithm for extracting handwritten text from express text based on Lab color space clustering. But only one kind of express text is selected, so this algorithm has great limitations. Yue Youjun et al [16] proposed a method of removing crop shadow in UVI space based on combining multi color channel. Through the combination of color channels, the shadows were removed in crop image. Zhong Xinxiu et al [17] proposed a threshold segmentation method based on Lab color space, which can filter the background of log image.

A segmentation method based on Lab color space was proposed for stained Tibetan ancient images which use location information of the three channels of Lab color space to obtain the stain's position information and weaken or eliminate the influence of stains. Then using the proposed method block image automatically, and perform the binarization process. Finally, the binarized image is obtained.

\section{Color space}

Most of the traditional image binarization methods are processed on gray scale images, but grayscale images contain much less information than color images. A lot of useful information is inevitably lost in the process of converting color image to gray image. So using the information of color images, we can achieve good binarization results. Common color space has RGB, HSI, Lab color space and so on, and color images have different expression forms in different color spaces. So using different color space conversion methods, we can get different available information to deal with different images and get the binarized format.

RGB color space. RGB color space is the most common color space, which is defined by CIE (International Illumination Committee) in 1931, and it is used in color shows of display system. RGB color space is divided into R (red), G (green), B (blue) three channels, the range of each channel is: R: 0-255, G: 0-255, B: 0-255. Any color in nature can be mixed by three components of the RGB color space, concrete realization way as shown in equation (1).

$$
F=r(R)+g(G)+b(B)
$$

Where $r(R), g(G), b(B)$ represents the size of red green and blue, when the three component is 0 (minimum), the mixture is black, and white when the three component is 255 (maximum).

Lab color space. Lab color space is a device independent color model defined by CIE (International Illumination Committee) in 1931, which is based on human perception of color and is closer to human vision. Lab color space is made up of brightness (L) and other two elements of color (a) and (b): the channel L range is [0,100], from black to white; the channel a range from green to gray to red, with a range of $[-128,127]$; the channel $b$ ranges from blue to gray to yellow, with range of $[-128,127][18]$.

The usual input images are RGB color images, and the image under the Lab color space needs to be transformed by RGB space, and an intermediate conversion process is required [11], concrete realization way as shown in equation (2-5). 


$$
\begin{aligned}
& \left(\begin{array}{l}
X \\
Y \\
Z
\end{array}\right)=\left(\begin{array}{lll}
0.607 & 0.174 & 0.200 \\
0.299 & 0.587 & 0.114 \\
0.000 & 0.066 & 1.116
\end{array}\right)\left(\begin{array}{l}
R \\
G \\
B
\end{array}\right) \\
& L=116\left(\sqrt[3]{\frac{Y}{Y_{0}}}\right)-16 \\
& a=500\left[\sqrt[3]{\frac{X}{X_{0}}}-\sqrt[3]{\frac{Y}{Y_{0}}}\right] \\
& b=200\left[\sqrt[3]{\frac{Y}{Y_{0}}}-\frac{Z}{Z_{0}}\right]
\end{aligned}
$$

Where $Y / Y_{0}>0.01, X / X_{0}>0.01, Z / Z_{0}>0.01,\left(X_{0}, Y_{0}, Z_{0}\right)$ is the standard white value.

\section{binarization algorithm}

OTSU. OTSU is a global threshold algorithm, based on the gray histogram of the image, the maximum variance between the target and the background is used as the threshold selection criterion, its basic ideas are as follows: suppose the gray level of the original image is L, the number of pixels which gray value equal to the $\mathrm{i}$ is $n_{i}$, the total number of pixels is $\mathrm{N}$, then the probability of occurrence of each gray level is: $p_{i}=n_{i} / N$; the gray level is divided into $C_{1}(1,2,3, \ldots, t)$ and $C_{2}(t+1, t+2, \ldots, L-1)$ according to the threshold $\mathrm{t}$, The probabilities of occurrence of $C_{1}$ and $C_{2}$ are: $p_{1}(t)=\sum_{i=0}^{t} p_{i}$ and $p_{2}(t)=\sum_{i=t+1}^{L-1} p_{i}$. So the mean values of $C_{1}$ and $C_{2}$ are:

$$
\begin{aligned}
& \mu_{1}(t)=\frac{\sum_{i=0}^{t} i p_{1}}{p_{1}(t)} \\
& \mu_{2}(t)=\frac{\sum_{i=t+1}^{L-1} i p_{i}}{p_{2}(t)}
\end{aligned}
$$

The gray average of the whole image is:

$$
\mu=\sum_{i=0}^{L-1} i p_{i}
$$

So the variance between classes is:

$$
\sigma_{B}^{2}(t)=p_{1}(t)\left[\mu_{1}(t)-\mu\right]^{2}+p_{2}(t)\left[\mu_{2}(t)-\mu\right]^{2}=p_{b}(t)\left[1-p_{b}(t)\right]\left[\mu_{1}(t)-\mu_{2}(t)\right]^{2}
$$

When $\sigma_{B}^{2}(t)$ maximum, $\mathrm{T}$ is:

$$
T=\operatorname{Arg} \underset{0 \leq t \leq L-1}{\operatorname{Max}} \sigma_{B}^{2}
$$

\section{NIBLACK}

NIBLACK is a local threshold algorithm, the basic idea is: first, the neighborhood size of pixels is defined, and then the threshold of the pixel $(\mathrm{x}, \mathrm{y})$ is calculated according to the mean and standard deviation of the pixel values in the neighborhood, as shown in the formula (11):

$$
T(x, y)=m(x, y)+k * s(x, y)
$$

Where $\mathrm{T}(\mathrm{x}, \mathrm{y})$ represents the threshold at the pixel point $(\mathrm{x}, \mathrm{y}), \mathrm{m}(\mathrm{x}, \mathrm{y})$ and $\mathrm{s}(\mathrm{x}, \mathrm{y})$ indicate neighborhood mean and standard deviation, respectively, and $\mathrm{k}$ is coefficient. 


\section{SAUVOLA}

SAUVOLA is an improvement to the NIBLACK, its effect is superior to NIBLACK in every aspect, the local threshold is calculated as follows:

$$
T=m *\left[1+k *\left(\frac{s}{R}-1\right)\right]
$$

Where $\mathrm{m}$ is the mean of the local pixel value, $\mathrm{s}$ is local variance, and $\mathrm{k}$ is empirical parameter, $\mathrm{R}$ is the dynamic range of the standard deviation.

\section{Our algorithm}

The idea of our algorithm is: first, convert the image from the RGB color space to the Lab color space. Based on Lab color space, weaken and eliminate the influence of the stain using the location information of the stain obtained by the information of the image itself. Then, the size of the block is automatically determined according to the number and size of the text in the image, so that the image can be automatically divided into blocks. Finally obtain the binarized image. The flow of our algorithm is shown in fig. 1.

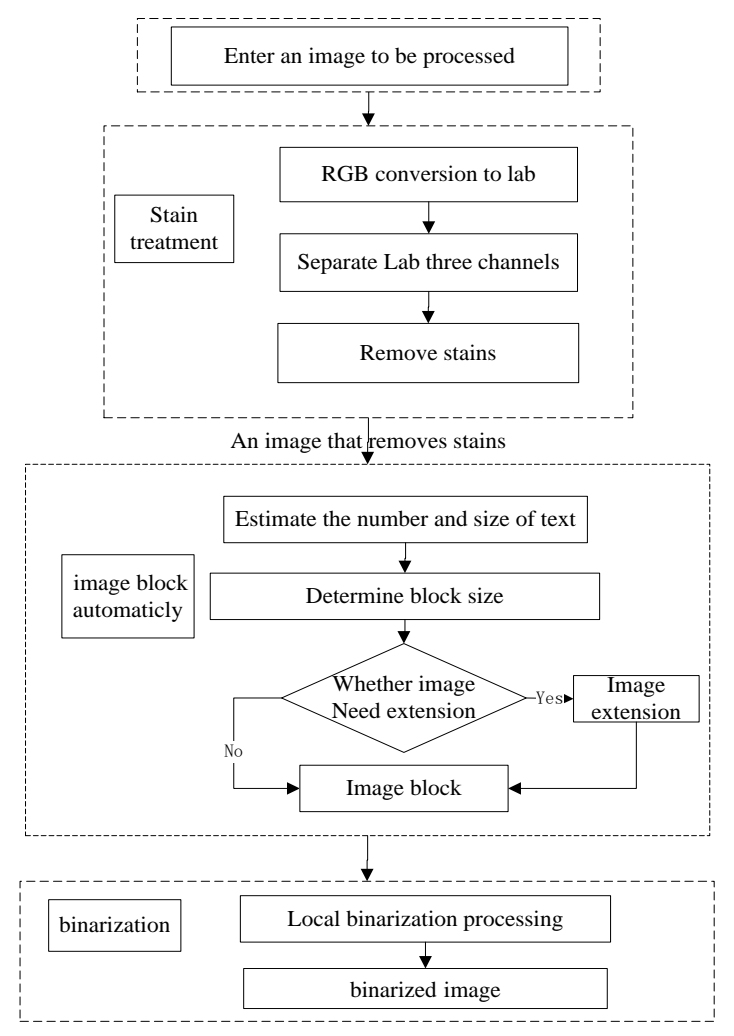

Fig. 1. The flow of our algorithm

\section{Deal with stains}

Channel separation. First, separate the three channels of the input image (fig. 2), the image of the three channels are displayed respectively, as shown in fig. 3, fig. 4, fig. 5.

Among them, the channel L is a brightness image, in the brightness range of $[0,100]$ to display the content of the color image, as we can see from fig. 3, the image under the channel a can better display the content of the original image in the form of gray image when there is no noticeable stain on the input image, the image quality under channel $\mathrm{L}$ is poor when there is noticeable stain on the input image; The image under channel a and channel $\mathrm{b}$ represent variations in color: The image under channel a represents color change from green to red, and the range is $[-128,127]$; The image under channel $b$ represents color change from blue to 
yellow, and the range is $[-128,127]$; In the color Tibetan ancient document image, the color of the stain is yellow, which is obviously different from the color of the text and background, as we can see from fig. 3, The image under channel b shows the difference of the color visually, highlighting the location of the blocks and smaller spots.

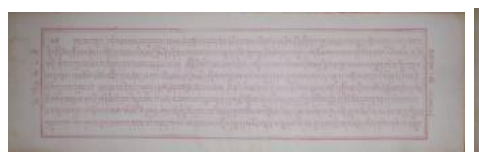

(a) Input image $\mathrm{A}$

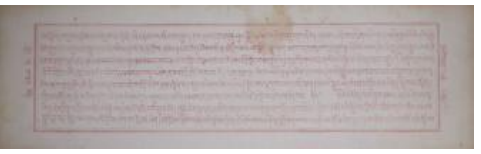

(b) Input image B

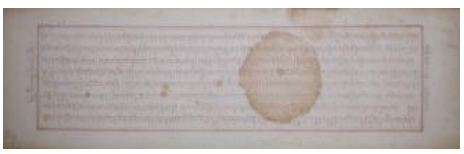

(c) Input image $\mathrm{C}$

Fig. 2. Three kinds of Tibetan ancient document image with stains

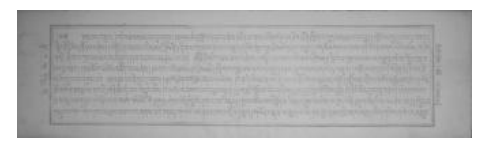

(a) Channel $\mathrm{L}$ of image $\mathrm{A}$

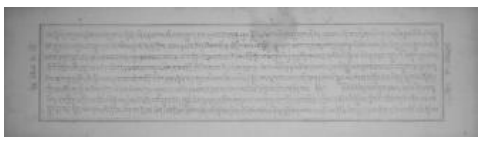

(b) Channel L of image B

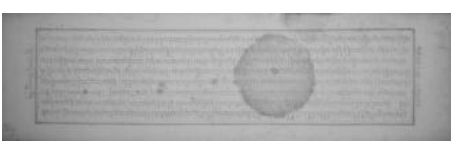

(c) Channel L of image C

Fig. 3. Image under channels L

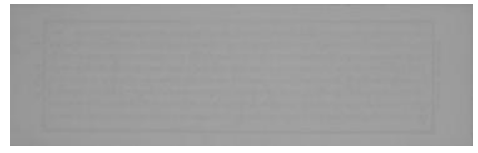

(a) Channel a of image $\mathrm{A}$

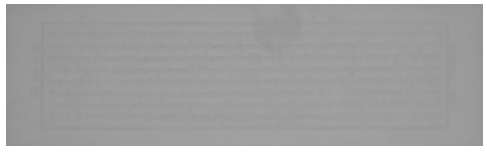

(b) Channel a of image B

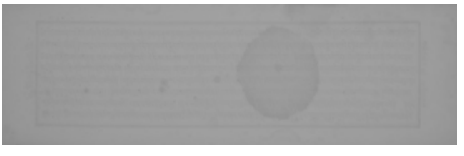

(c) Channel a of image $\mathrm{C}$

Fig. 4. Image under channels a

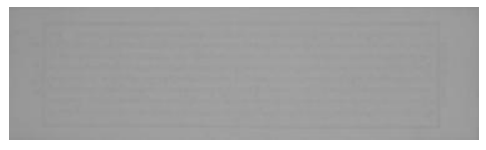

(a) Channel b of image $\mathrm{A}$

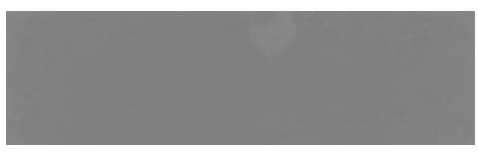

(b) Channel b of image $\mathrm{B}$

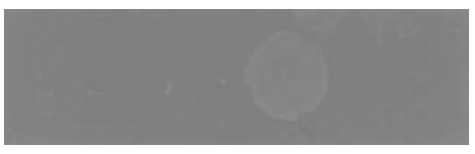

(c) Channel b of image $\mathrm{C}$

Fig. 5. Image under channels $b$

\section{Stain removal}

After many experimental tests and prior knowledge, we can see, the main reason stains are often mistaken for text is that the color of the stain area is similar to the color of the text in the color image, or there is a intersection between the stain and the gray value range of the text, which caused the stain to be judged as text.

The purpose of the stain treatment is to reduce the similarity between the stain and the text, and reduce the intersection of the range of the gray value of the text and the stain. The basic idea is to raise the pixel value of the dirty area and reduce the pixel value in the text region. According to the character of the Lab color space, the image under the channels a represents the change of color from green to red, and the range is [-128, 127], and in the Tibetan Ancient document image, the texts is exactly red. Therefore, the image under channels a not only does not help to reduce the pixel value of the text region, but will have the opposite effect. Based on the yellow color of the stain, we use image under channels $L$ and image under channels $b$ to deal with stains in the image.

Using the location information of the stains in the image under channels $b$, improved the pixel value of the dirty area in the image under channels $L$, the intersection of the text and the pixel value range is reduced or even disappeared, so as to reduce the influence of the dirt on the binarization's effect. In this process, the pixel value of the text area will be raised at the same time, but the pixel value of the text region will be increased slightly. In general, although the text pixel value is 
improved, it does not affect the processing effect. The processing results are shown in fig. 6 , specific calculation methods are shown in the formula (13):

$$
N I=m^{*} L+n * b
$$

Where, NI is the processed image, $\mathrm{L}$ is the image under channels $\mathrm{L}, \mathrm{b}$ is the image under channels $b, m$ and $n$ are coefficients, and the range is $[0,1]$.

The image under channels b highlights the stained area and blurs the text and background area, and the pixel value in the dirty area is greater than that of the text and background areas, The image under channels L contains all the information of text and stains, but the pixel values of the two are relatively close. Therefore, when choosing the coefficients, the weight of $n$ is increased so that the pixel values in the dirty areas are greatly improved, meantime the weight of $\mathrm{M}$ should be small. This is because when the weight of the $\mathrm{M}$ is too large, although the pixel value of the stain will be further increased, but will terminate at 255, the increase of the text pixel value will reduce the gap between the text pixel value and the dirty pixel value, that's not what we expected. The experimental results show that when the coefficient $\mathrm{m}$ and $\mathrm{n}$ are 0.4 and 0.9 respectively, the stain treatment effect is the best.

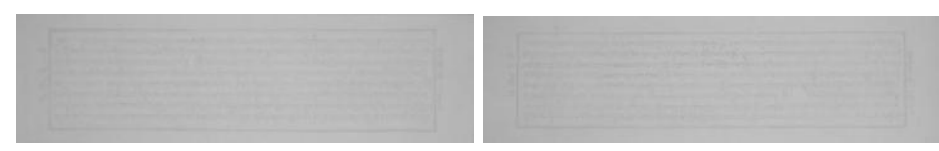

(a) Result of image A (b) Result of image B

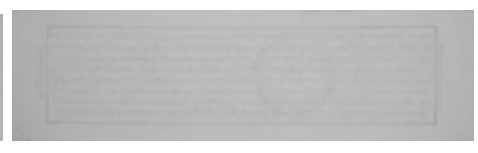

(c) Result of image C

Fig. 6. Stain treatment result

Automatic block of image. Experiments have proved that the block size of document images should be similar to or slightly larger than the area of a text box. This is because when the block is too large, the binarization effect will be similar to the global threshold method, which makes it easy to misjudge and cause the text information to be lost, but when the block is too small, the binarization effect is similar to the NIBLACK algorithm, cause false positives to produce noise points. Therefore, when the size of a block is similar to or slightly larger than the area of a rectangular box in which a text is taken, the calculated threshold size can separate the text better.

A method of automatic block segmentation based on the size of rectangular frame occupied by text is proposed, the basic idea is: First, the rows projection is used to get the number of rows, the location information of the rows and the length of the rectangular frame occupied by the text; Then a line of text is captured, and the width of the text rectangle and the estimated number of lines of text are obtained by column projection; Next the size of the rectangle box is calculated by the estimated value of the image area and the number of characters, and then the size of the block is obtained. Specific implementations are as follows: First, make a projection of the image after stain treatment, and sum up all the pixel values of each row according to formula (14), and show the result graphically, as shown in fig. 7: Compared to the background, the pixel value of the text is small, so the peaks in fig. 7 represent the background in the image, and the troughs represent the text lines in the image, the two troughs at the left and right represent the two horizontal lines at the upper and lower ends of the image.

$$
\operatorname{Sum}(i)=\sum_{j}^{N} f(i, j)
$$

Where Sum(i) represents the result of summing the values of row I pixels, $f(i, j)$ represents the size of the row $\mathrm{j}$ column pixel value in row $\mathrm{i}, \mathrm{N}$ represents the column number image.

According to the formula (14), statistics the number of troughs (text line) and gets information about where the text lines are located, calculate the width $\mathrm{X}$ of a line of text based on the positional information, and intercept a line of text in the image. The result is shown in fig. 9.

Similar to line projection principle, a vertical projection of a line of randomly intercepted text (fig. 9 ) is shown in fig. 8, The number of troughs is Num_Y (An estimate number of the text), and the 
location information of text is obtained. Finally, according to the area of the image and the estimated value of the number of characters in the image, the area of a rectangle box is calculated, and then the size of the block is obtained.

$$
\begin{aligned}
& S=M * N \\
& N u m=X * N u m_{-} Y \\
& \text { Per_S }=S / N u m \\
& \mathrm{Y}=\mathrm{Per}_{-} S / X
\end{aligned}
$$

Where $\mathrm{S}$ represents the area of the image, $\mathrm{M}$ and $\mathrm{N}$ represent the number of rows and columns of image, Num represents the estimate number of the words, Per_S represents the estimated area of each word, $\mathrm{X}$ and $\mathrm{Y}$ represents the size of the block.

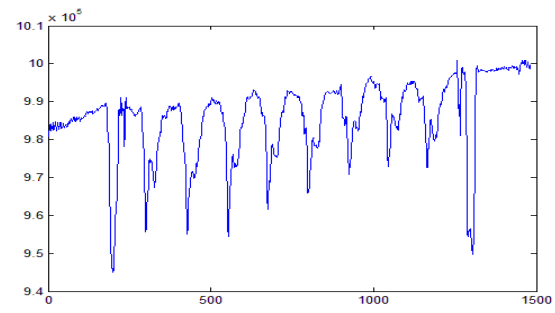

Fig. 7. Row projection

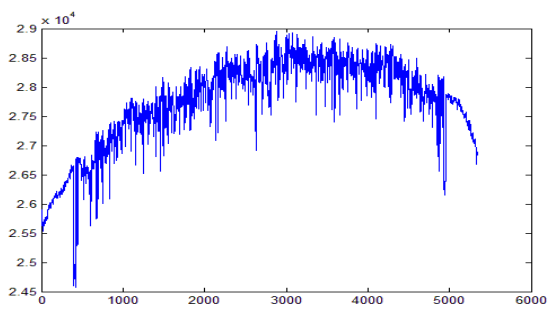

Fig. 8. column projection

Fig. 9. A line of text of processed image

\section{Binarization}

OTSU use one threshold for the whole image to get the binarized image, for the Tibetan Ancient document image, the processing effect is poor; NIBLACK is sensitive to its coefficient $\mathrm{K}$, and exit many false positive phenomena, lead to many noise spots in the binarized image; SAUVOLA is the improvement of NIBLACK, The parameter $\mathrm{R}$ is added, and the multiplication of $\mathrm{m}$ and $\mathrm{s}$ increases the influence of $\mathrm{s}$, while suppressing the influence of the coefficient. Experiments show that SAUVOLA in Tibetan ancient document image binarization processing is much better than OTSU and NIBLACK.

When an image is partitioned according to the size of the block calculated by automatic block division, the image size may not match the number of blocks, the image size should be mirrored according to the desired image size so that the image size can meet the requirements of blocking. In each block, the SAUVOLA is used for binarization processing ( $\mathrm{k}$ takes $0.5, \mathrm{R}$ takes 128), the local threshold is calculated in the block, then the text in the block is separated. When all the blocks are processed, the whole binarized image is obtained. The method of combining the global and the local has achieved good results in the binarization of Tibetan ancient document image.

\section{Results and discussion}

Result one. In order to verify the effectiveness of our method, three kinds of stains that may appear in Tibetan ancient document image are selected and processed. The three cases are small blocks of stains, large blocks of stains, and larger darker stains. The experimental res ults are as follows: 


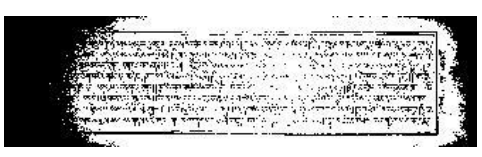

(a) Results of image A

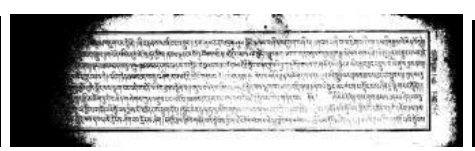

(b) Results of image B

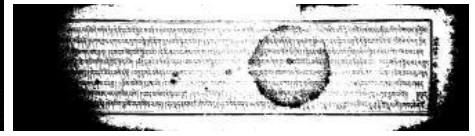

(c) Results of image $\mathrm{C}$

Fig. 10. Processed results of OTSU

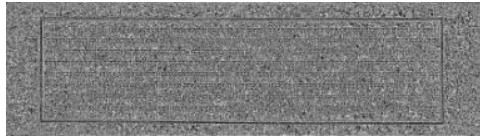

(a) Results of image $\mathrm{A}$

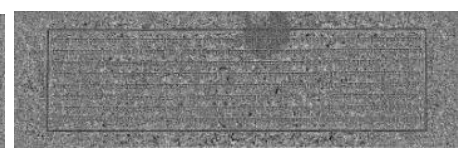

(b) Results of image B

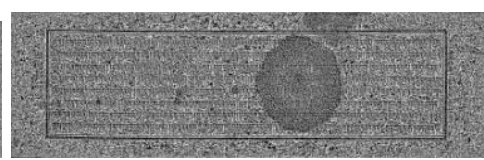

(c) Results of image $\mathrm{C}$

Fig. 11. Processed results of NIBLACK

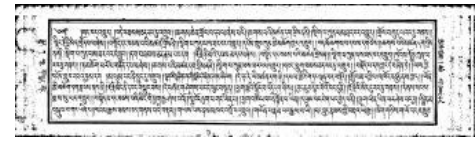

(a) Results of image $\mathrm{A}$

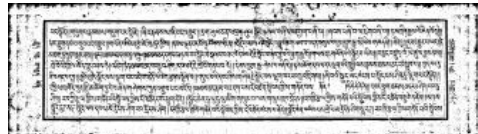

(b) Results of image B

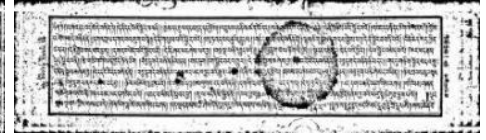

(c) Results of image $\mathrm{C}$

Fig. 12. Processed results of stain free treatment

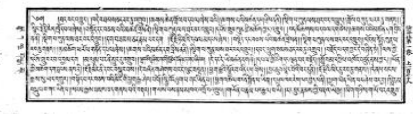

(a) Results of image $\mathrm{A}$

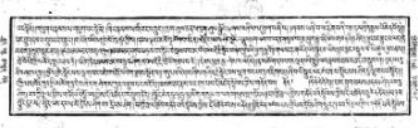

(b) Results of image B

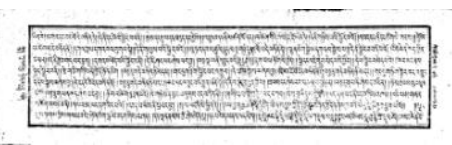

(c) Results of image C

Fig. 13. Processed results of our algorithm

As shown in fig. 10 and fig. 11, the traditional classical binarization algorithm(OTSU and ) is not suitable for the binarization processing of Tibetan ancient document image. Because in the target and the background area, the pixel value is not obvious and existing the stains, OTSU cannot accurately separate the target and background. NIBLACK excessive sensitivity to coefficients $\mathrm{K}$ and noise leads to too many false positives.

In Lab color space, different colors show great diversity. After channel separation, target and background have different forms in different channels. This difference can be used to eliminate the stains and separate the target very well, and a better processed results can be obtained. As we can see from fig. 13, our method has better effect on color Tibetan ancient document image, after binarization, the image is clear and the target details are well preserved. For stain areas that are not deep in color, the text is successfully extracted without damage. For the areas with darker stains, there are only a few false positives on the edges. Fig. 12 is the image which is not deal with stain, and the result is shown in fig. 12 and fig. 13, the method of combining the Lab color space with the stain treatment has a good effect on reducing and eliminating the stain.

Result two. In order to further prove the effectiveness of our method, a lot of images have been tested that the images have different quality and the size of the stain' s area is also different. The experimental results are as follows: 


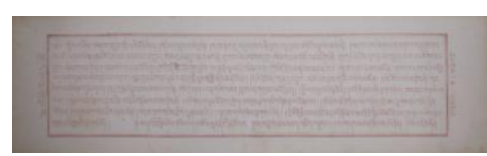

(a) Image $\mathrm{A}$

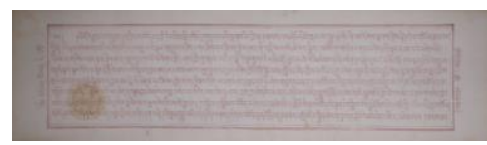

(d) Image D

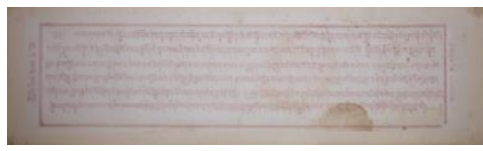

(g) Image G

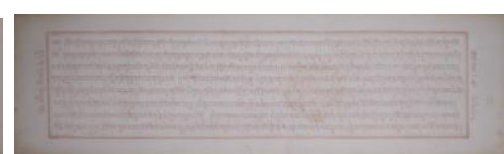

(b) Image $B$

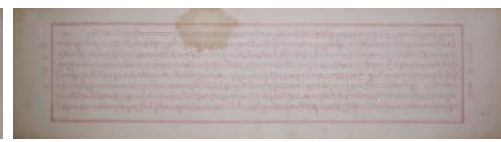

(e) Image $\mathrm{E}$

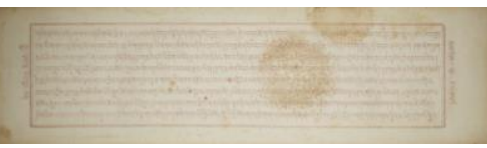

(h) Image $\mathrm{H}$

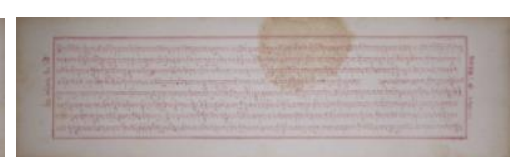

(c) Image $\mathrm{C}$

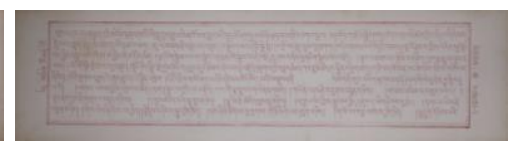

(f) Image F

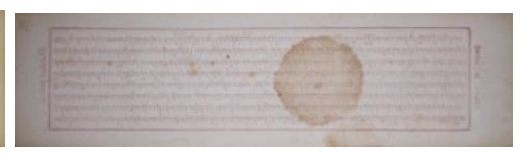

(i) Image I

Fig. 14. Different Tibetan ancient document images

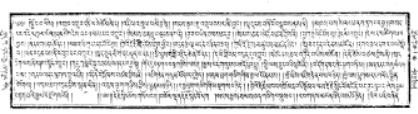

(a) Results of image A

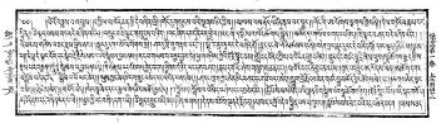

(d) Results of image D

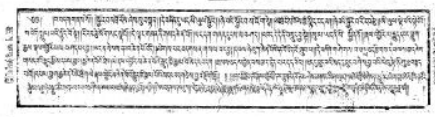

(g) Results of image $\mathrm{G}$

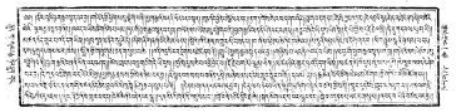

(b) Results of image B

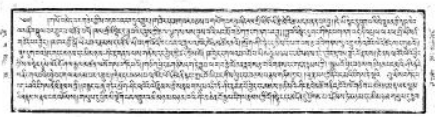

(e) Results of image E

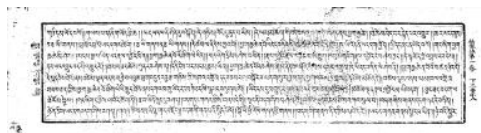

(h) Results of image $\mathrm{H}$

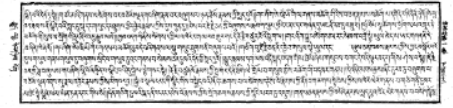

(c) Results of image $\mathrm{C}$

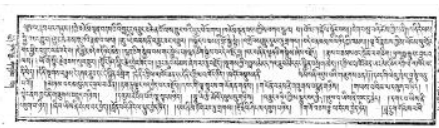

(f) Results of image F

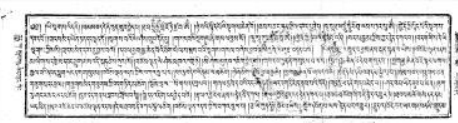

(i) Results of image I

Fig. 15. Processed results of our algorithm

As shown in fig. 14, there are nine stained Tibetan ancient document images. And the position and size of the stains are different in different image. A further examination was carried out to demonstrate the effectiveness of our method.

After a lot of experiments, the result we got further proof that our method has better binarization effect for stained Tibetan ancient document image. As shown in fig. 15, when the quality of the image is better or the stains in the image is not too darker, the text in the binarized image is clear and the influence of the stains in the image has been eliminated. When the quality of the image is not good or the stains in the image is too big and darker, the effect is not very satisfactory that a small amount outline of the stains are found in binarized image.

After experiments on a great many Tibetan ancient document images, it turns out to be the case that our method is very suitable for the binarization processing of Tibetan ancient document image. Although our method has some disadvantages that a small amount outline of the stains are found in binarized image for the image which the stains is too big and darker, but for the most of the Tibetan ancient document images binarization result is good, and the binarized image is very favorable for follow-up work. 
Result three. In addition to Tibetan ancient document image, we also experimented other document images using our method. The experimental results are shown in fig. 16 to fig. 23:

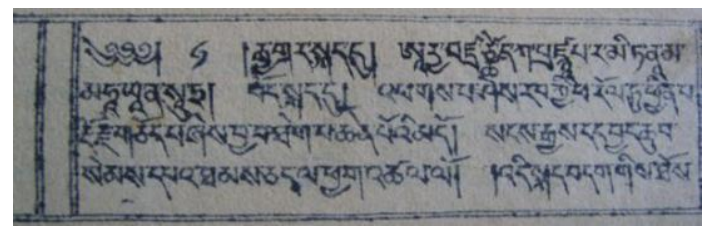

Fig. 16. Image J

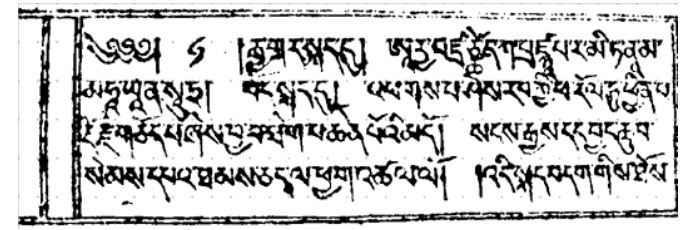

Fig. 17. Results of image $\mathrm{J}$

As shown in fig. 16 and fig. 17, this is another form of Tibetan ancient document image. Although its material and color are different from the other images we dealt with, its binarization results are still good. This is because the color difference between the foreground and the background is obvious, and our method mainly uses the differences between colors to get the binarized image.

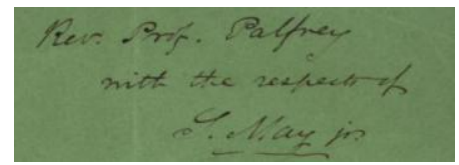

Fig. 18. Image $K$

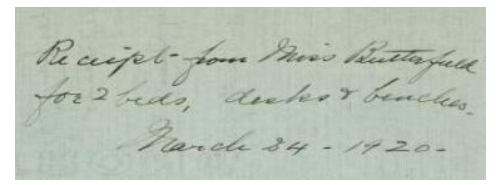

Fig. 20. Image L

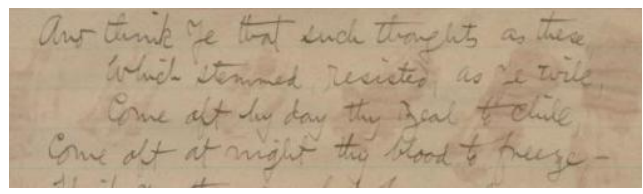

Fig. 22. Image $M$

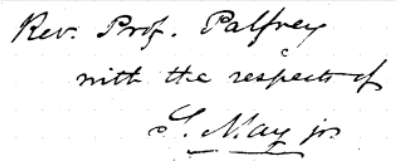

Fig. 19. Results of image $K$

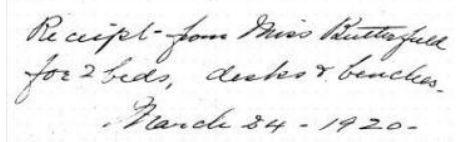

Fig. 21. Results of image $L$

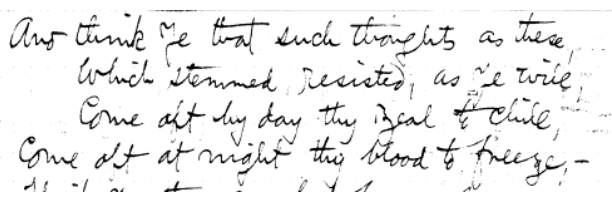

Fig. 23. Results of image $M$

As for fig. 18, fig 20 and fig. 22, these are three handwritten document images. Compared with the Tibetan ancient document images we dealt with, material and handwriting color are very different, but after binarization processing, we found that the binarization effect is good. As shown in fig. 19 and fig. 21, the text in the image is recognized clearly and the background separated very well. As for fig. 23, there are some noise points in binarized image ,this is because the stain in the fig. 22 is too darker. From these images, we can see that our method can be used not only for Tibetan ancient document images, but also for other types of images.

In conclusion, we can know from the results of these experiment, our method is very suitable for the binarization processing of Tibetan ancient document images. For this kind of Tibetan ancient document image, the color of the text, background and stains are different obviously. In Lab color space, using this difference we can eliminate the influence of stains, and then based on the size of rectangular frame occupied by text block image automatically, local binarization processing is performed using SAUVOLA algorithm, finally, binarized image was obtained.

Also, our approach have disadvantages, when the stain is too darker or the difference of color is not obvious, the binarization result is not good that there are noise points in the binarized image. 


\section{Conclusions}

In this paper, a local binarization method based on automatic segmentation of Lab color space is proposed to deal with the stain problem in ancient Tibetan document image. The method converts RGB images into three channel images in Lab color space firstly; Next, combining the image under the channel $\mathrm{L}$ and the image under the channel $\mathrm{b}$ to enhance the pixel contrast between the stain area and the target area, a grayscale image without stains is obtained; Then, the size of the image block is estimated according to the area occupied by the text, and the blocks are automatically divided; Finally, combining the SAUVOLA to get the binarized image. Experiments show that the method has a good binarization effect for the Tibetan ancient document image.

Our method mainly deals with Tibetan ancient document images with very large color difference. In lab color space, this difference will be enlarged, and through this property, we can eliminate the impact of stains. This method can be used not only for Tibetan ancient book with stains, but also for other images that there is a big difference in color. By using the color space property of Lab, the influence of noise on the target is eliminated, and the image is segmented better. Combinations of different channels will have different effects for different images, which will be some help for the another research. It is found that the method still has shortcomings, for the darker stain areas, there are some black spots at the edge in binarized image. How to eliminate these black spots needs to be solved step by step in future research.

\section{Acknowledgements}

This work was financially supported by the National Science Foundation (No.61375029 and No. 61772430), the personnel training program of State Ethnic Affairs Commission, and also funded by the Fundamental Research Funds for the Central University of Northwest Minzu University (No.31920170142), and the Discipline Construction Funds for "An Excellent and Three Specia" of Northwest Minzu University (No.10018702).

\section{References}

[1] Feng Yan: Research status of images binarization about Tibetan Ancient document image, Computer knowledge and technology Vol. 12 (2016), p. 144-146.

[2] Meng Lina, Han Qirui: An improved binarization method combined global thresholding with local thresholding, Computer Technology and Development Vol. 22 (2012), p. 116-119.

[3] Nobuyuki Otsu: A threshold selection method from gray -level histogram, IEEE Trans. Systems, Man, and Cybernetics Vol. 1 (1979), p. 62-66.

[4] Zhang Yifan, Kang Yan, Lin Ying: Image segmentation algorithm based on local dynamic threshold, Software Guide Vol. 12 (2013), p. 46-48.

[5] AMA Talab, Zhang Canhuang, Wang Junfei: An enhanced Bernsen algorithm approaches for vehicle logo detection, International Journal of Signal Processing, Image Processing and Pattern Recognition Vol. 7 (2014), p. 203-210 .

[6] Wang Xuzhe: Research on local adaptive binarization method, Software Guide Vol. 10 (2013), p. 13-14.

[7] J. Sauvola, M. PietikaKainen: Adaptive document image binarization, Pattern Recognition Vol. 33 (1999), p. 225-236.

[8] Madhuri Latha G, Chakravarthy G, An improved Bernsen algorithm Approaches for license plate recognition, Journal of Electronics and Communication Engineering Vol. 3 (2012), p. 1-5.

[9] W Junfei, H Zhagnchan, TALAB AMA, New binarization method called BM aim to optimize detail of image, Journal of Wuhan University of Technology Vol. 36 (2014), p. 127-132. 
[10] Lin Kaiyan, Wu Junhui, Xu LiHong, Overview of color image segmentation methods, Journal of Image and Graphics Vol. 10 (2005), p. 1-10.

[11]H.D. Cheng, X.H. Jiang, Y. Sun, Jingli Wang: Color image segmentation:advances and prospects, Pattern Recognition Vol. 34 (2001), p. 2259-2281.

[12] Lv Yanna, Zhu Xiao, Zhu Changhong: Common color space and its transformation in computer digital image processing, Computer and digital engineering Vol. 34 (2006), p. 54-56.

[13] Yang Jing, Zhu Lei: Color image segmentation method based on RGB color space, Computers and modernization (2010), p. 147-149.

[14] Pang Xiaomin, Min Zijian, Kan Jiangming: Color image segmentation based on HSI and LAB color space, Journal of Guangxi University: Natural Science Edition Vol. 36 (2011), p. 976-980.

[15] Jiang Jichun, Wang XiaoHong, Xu Qinrong: Research of handwritten text extraction algorithm based on Lab color space, Packaging Engineering Vol. 35 (2014), p. 139-143.

[16] Yue Youjun, Zhao Wenjia, Zhao Hui, Wang Hongjun: Method for removing crop shadow in UVI space based on multi color channel combination, Jiangsu Agricultural Sciences Vol. 45 (2017), p. 196-200.

[17]Zhong Xinxiu, Jing Lin, Lin Yaohai: Log-end Area Recognition Based on Lab Color Space and Threshold Segmentation, Journal of Longyan University Vol. 35 (2017), p. 95-99.

[18] Yang Biao, Yang Qin: A two vehicle license plate localization method based on Lab color space and morphological processing, Science, technology and Engineering Vol. 14 (2014), p. 108-110. 